\title{
Characteristics and Prevalence of Latent Autoimmune Diabetes in Adults (LADA)
}

\author{
Priyanka P. Brahmkshatriya, ${ }^{1}$ Anita A. Mehta, ${ }^{2}$ Banshi D. Saboo, ${ }^{3}$ and Ramesh K. Goyal ${ }^{4}$ \\ ${ }^{1}$ Department of Pharmacology, L. J. Institute of Pharmacy, Sarkhej Gandhinagar Highway, Gujarat, Ahmedabad 380015, India \\ ${ }^{2}$ Department of Pharmacology, L. M. College of Pharmacy, Navrangpura, Gujarat, Ahmedabad 380009, India \\ ${ }^{3}$ Department of Endocrinology, Dia-Care Clinic, 2 Gandhi Park, Nehrunagar, Gujarat, Ahmedabad 380015, India \\ ${ }^{4}$ Department of Pharmacology SPP School of Pharmacy \& Technology Management, Mumbai 400056, India
}

Correspondence should be addressed to Priyanka P. Brahmkshatriya, its_piks@yahoo.com

Received 27 November 2011; Accepted 9 January 2012

Academic Editors: S. Cuzzocrea and K. Wada

Copyright ( 2012 Priyanka P. Brahmkshatriya et al. This is an open access article distributed under the Creative Commons Attribution License, which permits unrestricted use, distribution, and reproduction in any medium, provided the original work is properly cited.

\begin{abstract}
Diabetes, one of the most commonly seen metabolic disorders, is affecting a major area of population in many developing as well as most of the developed countries and is becoming an alarming concern for the rising cost of the healthcare system. Latent Autoimmune Diabetes in Adults (LADA) is a form of diabetes which is less recognized and underdiagnosed type of diabetes which appears to have characteristics of both type 1 (autoimmune in nature) and type 2 diabetes (adult age at onset and initial response to oral hypoglycemic agents). An epidemiological study was carried out on 500 patients in the western region of India. Various parameters such as age at onset, duration of diabetes, gender, basal metabolic index (BMI), type of diabetes, family history, HbA1c levels, cholesterol levels, and current treatment regimen were evaluated and correlated with type 1 and type 2 diabetes. Moreover, diagnostic markers for LADA, namely, GAD autoantibodies and C-peptide levels, were determined for 80 patients selected from the epidemiological study. Some of the results obtained were found to be consistent with the literature whereas some results were found to be contradictory to the existing data.
\end{abstract}

\section{Introduction}

Diabetes mellitus (DM) includes a group of carbohydrate metabolism disorders which is characterized by hyperglycemia and leads to long-term macrovascular and microvascular complications. The prevalence of diabetes mellitus is increasing significantly in most of the developed and many developing countries, and it is of great concern $[1,2]$. The worldwide prevalence of diabetes mellitus has risen dramatically over the past two decades. It is one of the most common chronic endocrine disorders affecting millions of people worldwide. The International Diabetes Federation (IDF) data indicates that by the year 2025, the number of people affected by diabetes will reach 333 million. Although all forms of diabetes are characterized by hyperglycemia, the pathogenic mechanisms by which hyperglycemia arises differ widely. American Diabetes Association categorized DM mainly as types 1 and 2 diabetes and the others [3].
Individuals with type 1 diabetes show extensive beta-cell destruction, and therefore no residual insulin secretion, requiring insulin for survival. Autoimmune $\beta$-cell destruction is the main cause of insulin deficiency in type 1 diabetes [4]. In some individuals with diabetes, adequate glycaemic control can be achieved with weight reduction, exercise, and/or oral agents. These individuals, that is, patients of type 2 diabetes, therefore, do not require insulin and may even revert to impaired glucose tolerance (IGT) or normoglycaemia [5]. It is mainly a heterogeneous disease with a complex pattern of inheritance. Type 2 diabetes is the commonest form of diabetes constituting about $90 \%$ of the total diabetic population, whereas type 1 diabetes constitutes about $10-15 \%$ of the diabetic population. Geographic, environmental, and genetic factors all play a major role in the variation of incidence of all types of diabetes. Although diabetes is classified into two major types-type 1 (insulin dependent) and type 2 (insulin independent) diabetes, there 
are some forms of diabetes which cannot be classified into either of these categories. One such less recognized and underdiagnosed manifestation of DM appears to affect adults with many characteristics of type 2 diabetes and carries a high risk of insulin dependency progression, the condition known as Latent Autoimmune Diabetes in Adults (LADA) [3]. The usual features of LADA patients reported are onset of diabetes at $\geq 25$ years of age, clinical presentation masquerading as nonobese type 2 diabetes, unlikely to have a family history of type 2 diabetes, initial control of hyperglycemia with diet and oral antidiabetic agents, evolution to insulin necessity within months, and some features of type 1 diabetes such as low fasting $C$-peptide and positive GAD auto antibodies [6-8]. About $20 \%$ of the patients diagnosed with type 2 diabetes may have LADA. This accounts for 5-10\% of the total diabetes population, the same number as type 1 diabetes [9]. Despite the frequency of LADA, there are no universal recommendations regarding testing for islet antibodies in adult onset diabetes. A reliable clinical strategy is required to identify which adults with diabetes have a high likelihood of LADA and need testing for islet antibodies. Another important characteristic of LADA which faces controversy is the family history. Some reports suggest that LADA patients are unlikely to have family history of type 2 diabetes [10] while a recent study (NordTrøndelag Health Study) indicated strong evidence of family history as an important risk factor for LADA [11]. India is witnessing an epidemic of diabetes, more specifically type 2 diabetes. It has been observed that the onset of type 2 diabetes occurs at an early age in Asian population. Very less work has been done in context to LADA in the Indian population. Since it has been observed that the prevalence of diabetes in India and especially in Gujarat or Western population is significantly high, it follows that the prevalence of LADA in this population can be predicted to be high. Also, from the above reports, it is obvious that the differences between type 1 diabetes and LADA are not very well understood. Similarly, due to certain characteristics of LADA which phenotypically resemble type 2 diabetes, many LADA patients are often initially misdiagnosed as type 2 diabetic patients. Since not much work has been done in the field of LADA in India, where diabetes affects a significant sector of the society, study of prevalence, characteristics and immunological markers of LADA, especially in the Western (Gujarat) population, where the prevalence of diabetes is high, would help in providing valuable insights into the underlying molecular and genetic mechanisms, similarities or differences with other types of diabetes and designing appropriate diagnostic criteria and treatment regimen for such patients.

\section{Methods}

2.1. Epidemiological Study. The epidemiological study was carried out on 500 patients randomly selected, having any type of diabetes (excluding prediabetic syndrome or IGT) as per the Study Protocol approved by the Independent Ethics Committee Medilink Research Centre, Medilink Hospital, Ahmedabad, India.
2.1.1. Patient Recruitment and Screening. Patients attending DIACARE Clinic (Ahmedabad) and Medilink Hospital (Ahmedabad) confirmed as diabetic as per WHO criteria [12] were included in the epidemiological study. Patients not having diabetes or who are seriously ill were excluded from the study. All patients were given a patient information sheet and an informed consent form. Blood $(10 \mathrm{~mL})$ was collected for determination of various biochemical parameters.

2.1.2. Study Procedure. Various parameters evaluated for the epidemiological study were present age; age at onset of diabetes; duration of diabetes; gender; basal metabolic index (BMI); type of diabetes; family history of diabetes, if any; diet and lifestyle; medical complications; cholesterol levels; HbA1c levels; the current treatment regimen that the patient was following.

\subsection{Estimation of C-Peptide Levels ( $\beta$-Cell Function) and GAD Autoantibodies}

2.2.1. Patient Recruitment. Eighty diabetic patients from the epidemiological study, attending DIACARE Clinic, and Medilink Hospital, Ahmedabad, were selected and recruited after checking the required inclusion and exclusion criteria. These patients were grouped into the four categories according to their type of diabetes: type-1 diabetes $(n=20)$; type 2 diabetes $(n=20)$; LADA suspects $(n=20)$; and patients newly diagnosed with diabetes and who could not been assigned a particular class of diabetes $(n=20)$.

Patients who were seriously ill or who had any specific objection for undergoing the tests for estimation of C-peptide and GAD auto antibodies were excluded from the study.

2.2.2. Determination of C-Peptide Levels. C-peptide levels were determined in the above groups using the EiAsy Way diagnostic kit (Diagnostic Biochem Canada Inc., Canada). The kit was used for the indirect quantitative determination of C-peptide by Enzyme Linked Immunosorbant Assay (ELISA) in human serum in vitro [13].

2.2.3. Estimation of GAD Autoantibodies. GAD autoantibodies were determined in the above-mentioned groups using Isletest GAD diagnostic kit (Biomerica, USA). The kit was used for an in vitro qualitative ELISA test for detection of circulating autoantibodies against GAD antigens [14].

2.3. Statistical Analysis. After recording the details of patients, the data was sorted into different groups according to the type of diabetes (type 1, type 2, LADA, and undiagnosed). The mean values were calculated for each parameter and compared in different groups. All results were expressed as mean \pm SEM or as percentage. The statistical differences between various groups were evaluated by One Way Analysis of Variance (ANOVA) followed by Tukey's Test or Chi-Square Test with Yate's Correction (for nonparametric data). Data were considered significant at $P<0.05$ and highly significant at $P<0.001$. Statistical analysis was performed using GraphPad statistical software. 
TABLE 1: Comparative evaluation of different parameters in different types of diabetes including LADA.

\begin{tabular}{|c|c|c|c|c|}
\hline Parameter & Type 1 & Type 2 & LADA & Undiagnosed \\
\hline Prevalence $(\%)$ & 6.2 & 79.2 & 5 & 9.6 \\
\hline \multicolumn{5}{|l|}{ Gender-wise prevalence } \\
\hline Males (\%) & 38.7 & 62.12 & 80 & 54.16 \\
\hline Females $(\%)$ & 61.3 & 37.87 & 20 & 45.83 \\
\hline Average age (yrs) & $21.64 \pm 1.97$ & $55.69 \pm 0.47$ & $40.8 \pm 1.48$ & $44.46 \pm 1.56$ \\
\hline Range (yrs) & 6-59 & $33-82$ & $23-55$ & $25-65$ \\
\hline Mean age at onset of diabetes (yrs) & & $48.01 \pm 0.5$ & $33.4 \pm 2.15$ & $37.68 \pm 1.28$ \\
\hline Range (yrs) & $2-30$ & $30-75$ & $22-44$ & $20-45$ \\
\hline Prevalence in different age groups (\%) & $\mathrm{M}$ & $\mathrm{M} \quad \mathrm{F}$ & $\mathrm{M}$ & M \\
\hline$<12$ years & 16.66 & 0 & 0 & 0 \\
\hline $12-24$ years & 41.66 & 0 & 0 & 0 \\
\hline $25-35$ years & 16.66 & 0.8 & 15 & 36.36 \\
\hline $36-45$ years & 16.66 & 15.04 & 20 & 30.76 \\
\hline$>45$ years & 8.33 & 84.14 & 20 & 57.69 \\
\hline \multicolumn{5}{|l|}{ Family history (\% patients) } \\
\hline Present & 35.48 & 57.07 & 52 & 70.81 \\
\hline Absent & 65.42 & 42.93 & 48 & 29.17 \\
\hline Basal metabolic index (BMI, \% patient & & & & \\
\hline Underweight (U) & 38.6 & 1.76 & 12 & 5 \\
\hline Healthy $(\mathrm{H})$ & 58 & 25.5 & 76 & 55 \\
\hline Overweight (H) & 3.2 & 47.3 & 12 & 35 \\
\hline Obese $(\mathrm{O})$ & 0 & 22.9 & 0 & 5 \\
\hline Very obese (VOb) & 0 & 2.27 & 0 & 0 \\
\hline Mean BMI & $19.96 \pm 0.629$ & $27.95 \pm 0.26$ & $22 \pm 0.55$ & $25.68 \pm 0.65$ \\
\hline BMI range & $13.75-25$ & $16-45$ & $15-26$ & $16.5-34$ \\
\hline Treatment regimen (\% patients) & & & & \\
\hline Insulin & 92.5 & 3.5 & 20 & 12 \\
\hline OHA & 3.5 & 65.5 & 16 & 53.5 \\
\hline Insulin + OHA & 3.5 & 29.5 & 60 & 34.5 \\
\hline Drug therapy (\% patients) & & & & \\
\hline $1 \mathrm{DT}$ & 96.4 & 18 & 23.6 & 28 \\
\hline $2 \mathrm{DT}$ & 3.6 & 28 & 23.8 & 28 \\
\hline $3 \mathrm{DT}$ & 0 & 29 & 23.8 & 12.5 \\
\hline $4 \mathrm{DT}$ & 0 & 21.5 & 28.5 & 22 \\
\hline $5 \mathrm{DT}$ & 0 & 3 & 0 & 9.4 \\
\hline Mean HbA1c levels (\%) & $9.661 \pm 1.10$ & $8.21 \pm 0.25$ & $10.45 \pm 0.75$ & $7.89 \pm 0.47$ \\
\hline Mean cholesterol levels $(\mathrm{mg} / \mathrm{dL})$ & No data available & $181.96 \pm 5.28$ & $222.33 \pm 26.87$ & $210.90 \pm 13.59$ \\
\hline Mean C-peptide levels (ng/mL) & $0.36 \pm 0.05$ & $1.26 \pm 0.36$ & $0.34 \pm 0.05$ & $0.63 \pm 0.15$ \\
\hline GAD autoantibodies & $0.76 \pm 0.05$ & $0.7 \pm 0.06$ & $0.95 \pm 0.06$ & $1.01 \pm 0.07$ \\
\hline (units/mL) & Absent & Absent & Present & Present \\
\hline
\end{tabular}

\section{Results and Discussion}

Results of different parameters evaluated amongst different classes of diabetic patients are summarized in Table 1.

According to reports, type 2 diabetes constitutes a major section, that is, $80-90 \%$ of the total diabetic population [3]. Furthermore, about $20 \%$ of the patients diagnosed with LADA may have type 2 diabetes. This accounts for $5-10 \%$ of the total diabetes population, the same number as type 1 diabetes [9]. The results of the present study in a diabetic population revealed that the prevalence of type 2 diabetes was the highest (79.2\%), followed by type 1 diabetes $(6.2 \%)$ and LADA (5\%) being nearly equal. This implies that the findings are in accordance with the reported data. Another group, comprising of patients who could not be categorized into one particular class of diabetes constituted of around $10 \%$ of the total diabetic population.

The prevalence of diabetes among males and females varies with geographical location, ethnicity, genetic and environmental factors (Figure 1). 


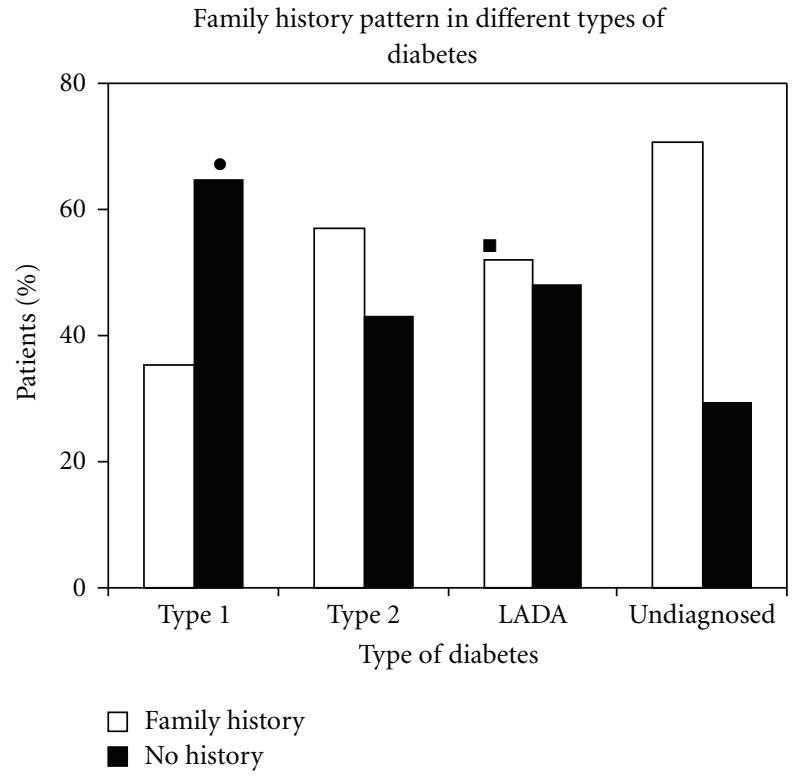

Figure 1: Prevalence of different types of diabetes in males and females. Black circle: prevalence pattern significantly different in type 1 diabetes as compared to type 2 diabetes $(P<0.01)$. Black square: prevalence pattern significantly different in LADA as compared to type 1 diabetes $(P<0.01)$.

According to the global prevalence of diabetes studies, overall, diabetes prevalence is higher in men than in women [15]. The results of the present study revealed that diabetes was found to be more prevalent in men (61\%) than in women (39\%). Thus the findings are in accordance with the reported data $[15,16]$. Further studies on prevalence of different types of diabetes in males and females revealed that there was a significant difference in the prevalence pattern of diabetes in males and females in LADA and type 1 diabetes $(P<0.01)$. Type 1 diabetes was found to be more prevalent in females $(61.3 \%)$ than in males $(38.7 \%)$, whereas the prevalence of LADA was significantly more in males $(80 \%)$ than in females $(20 \%)$. This suggests that males might be more prone towards development of LADA than females. There was a significant difference in prevalence pattern of type 1 and type 2 diabetes $(P<0.01)$. The prevalence pattern of type 2 diabetes, LADA, and undiagnosed patients was similar with no significant differences. Amongst type 2 diabetes patients, $62.12 \%$ were males and $37.87 \%$ were females. The similar prevalence pattern was seen in undiagnosed patients, that is, $54.16 \%$ males and $45.83 \%$ females suggesting that these patients are likely to be type 2 or LADA.

Studies showed that the average age in different diabetic populations varied significantly. The average age of LADA $(40.88 \pm 1.48$ years $)$ patients was found to be significantly lower than type 2 diabetes and significantly higher than type 1 diabetes $(P<0.01)$. The average age of patients of type 1 diabetes $(21.64 \pm 1.97$ years $)$ was found to be significantly lower than type 2 and LADA $(P<0.01)$. The average age of patients with type 2 diabetes $(55.69 \pm 0.47$ years) was the highest as compared to previous two groups. The average age in undiagnosed patients $(44.46 \pm 1.56$ years $)$ suggested that

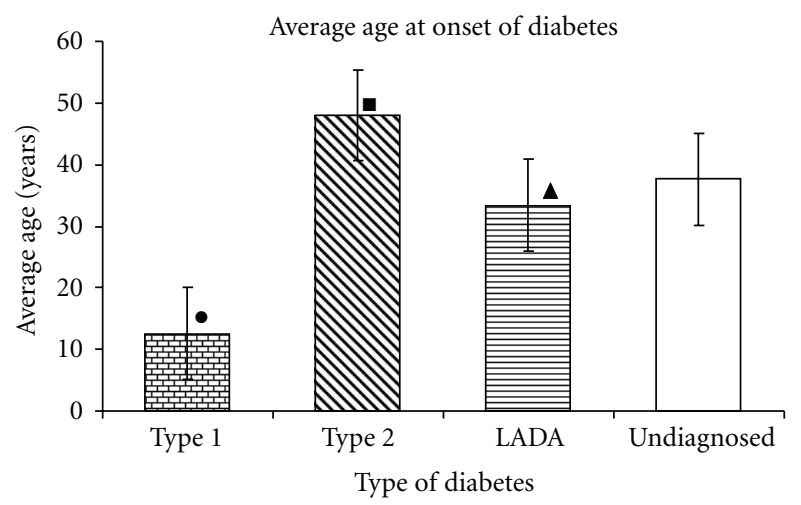

Figure 2: Average age at onset of different types of diabetes. Black circle: significantly lower age of onset in type 1 diabetes compared to type 2 diabetes $(P<0.001)$ and undiagnosed patients $(P<0.001)$. Black square: significantly higher age of onset in type 2 diabetes compared to undiagnosed patients $(P<0.001)$. Black triangle: significantly lower age of onset in LADA compared to type 2 diabetes $(P<0.001)$ and higher than Type $1(P<0.001)$.

these patients are more likely to be patients with LADA than that with type 2 diabetes.

The average age at onset is a very critical factor in determining the type of diabetes as well as the treatment regimen to be decided for the patient. According to reported data, one of the important identifying characteristics of LADA is adult age at onset ( $>25$ years) [6]. The present studies showed that the average age at onset of diabetes in patients with LADA $(33.4 \pm 2.15$ years $)$ was significantly higher than patients with type 1 diabetes patients $(12.55 \pm 1.34$ years $)(P<$ 0.001 ) and significantly lower than patients with type 2 diabetes $(48.01 \pm 0.50$ years $)(P<0.001)$. The mean age at onset in undiagnosed patients was observed to follow the same pattern (37.68 \pm 1.28 years) strongly suggesting that these patients are more likely to be patients of LADA than type 2 diabetes. Results are presented in Figure 2.

Further studies regarding the prevalence of diabetes in different age groups revealed that type 1 diabetes was found to be most frequently occurring in the age group of 12-24 years in both males and females. Similarly type 2 diabetes was found to be most prevalent in males and females above 45 years of age. Interestingly LADA was found to occur most frequently in males of 36-45 years of age, while it was seen to affect most females above 45 years of age. This finding may lead to an assumption that LADA might be affecting females at a higher age than males.

The impact of family history of diabetes (FHD) on LADA is less well understood than those for type 1 and type 2 diabetes [17]. Studies indicate that LADA has the same genetic features characteristic of type 1 diabetes, including an increased frequency of HLADQB1 genotypes [18, 19]. On the other hand, results from a British study indicated that $33 \%$ of patients with LADA have relatives with type 2 diabetes [20]. These findings suggest that LADA may share inherited features with both type 1 and type 2 diabetes [11]. Results of family history in different types of diabetes revealed significant differences in the family history pattern of LADA 


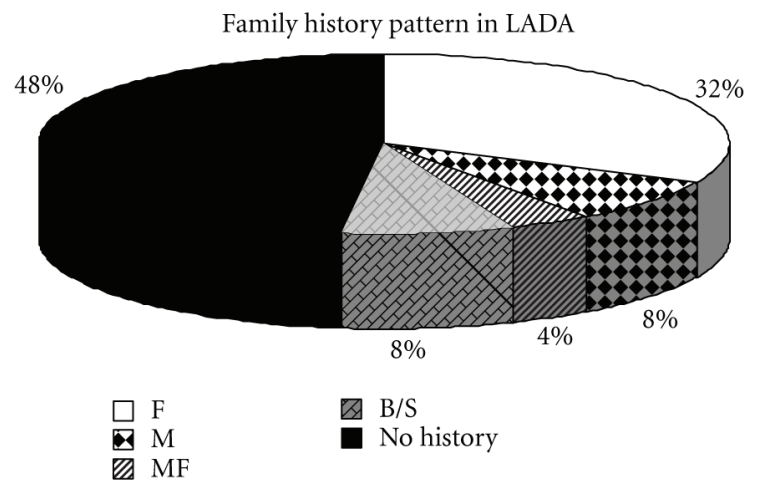

FIgUre 3: Family history pattern in LADA. F-Father, M-Mother, MF-Mother and Father, B/S- Brother or Sister.

and type 1 diabetes $(P<0.05)$. While family history was absent in majority of patients with type $1(65.42 \%)$, type 2 $(57 \%)$, LADA (52\%) as well as undiagnosed patients (70\%) showed significant presence of family history. The pattern seen in undiagnosed patients was significantly different than in type 1 diabetes $(P<0.01)$. There was a significant difference in family history patterns of type 1 and type 2 diabetes $(P<0.01)$. Absence of family history in patients with type 1 suggests that $\beta$-cell destruction in type 1 diabetes might be due to viral infections or triggered autoimmune mechanisms. The noticeable presence of family history in type 2 diabetes is in accordance with the fact that type 2 diabetes is strongly hereditary in nature. Among LADA patients, a significant population (32\%) showed paternal inheritance while a small proportion (8\%) showed maternal inheritance, sibling inheritance (8\%) and $4 \%$ showed both paternal and maternal inheritance. The results are shown in Figure 3.

Some reports suggest that LADA patients are unlikely to have a family history of type 2 diabetes [10], while some indicate presence of family history as an important risk factor for the development of LADA [11]. The interesting observation obtained in the present study would possibly open new doors for future exploration regarding pattern of inheritance in LADA patients.

In an effort to correlate BMI values with the type of diabetes, it was observed that a majority of patients with type 1 diabetes were healthy (58\%) or underweight $(38.6 \%)$ in nature suggesting possible alteration of carbohydrate, lipid, and protein metabolisms due to absolute insulin deficiency (Figure 4). The BMI values of patients with type 1 diabetes $(19.96 \pm 0.629)$ were significantly lower than patients with type 2 diabetes $(27.95 \pm 0.26)(P<0.001)$. BMI values of patients with type 2 diabetes showed that most of the patients of this category were overweight $(47.3 \%)$. Significant proportions of these patients were found to be obese $(22.9 \%)$ and very obese $(2.27 \%)$. This implies that the findings are in agreement with the existing literature which states that obesity is one of the prime factors leading to development of type 2 diabetes and insulin resistance [21]. Furthermore, the BMI values of LADA patients $(22 \pm 0.55)$ were significantly lower than patients with type 2 diabetes $(P<0.001)$ confirming the nonobese nature of these patients. None of

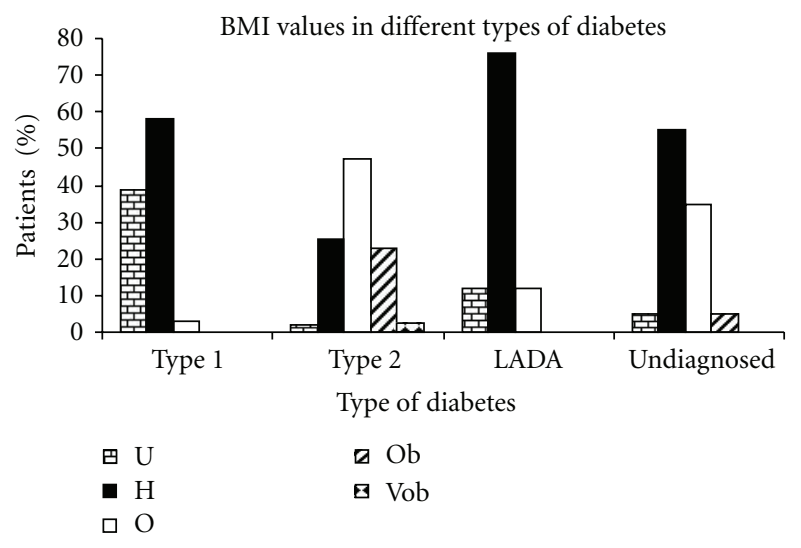

FIGURE 4: BMI values in different types of diabetes. U-Underweight, H-Healthy, O-Overweight, Ob-Obese, Vob-Very Obese.

the patients were obese which rules out the involvement of obesity and insulin resistance in development of LADA. Most of the patients $(76 \%)$ displayed BMI values corresponding to healthy individuals suggesting that the gradual $\beta$-cell destruction in LADA is insufficient to cause significant weight loss. Reports suggest that BMI values are lower in LADA patients compared to patients with type 2 diabetes [22]. Thus our findings were in accordance with the reported data. The mean BMI levels in undiagnosed patients $(25.68 \pm 0.65)$ were significantly lower than patients with type 2 diabetes $(P<0.05)$ and higher than patients with type 1 diabetes $(P<$ $0.001)$. A majority of the undiagnosed patients $(55 \%)$ were found to be healthy, while a noticeable proportion $(35 \%)$ was found to be overweight, though the proportion of overweight patients $(35 \%)$ was relatively less in undiagnosed patients than that of type 2 diabetics $(47.3 \%)$ suggesting that these patients are likely to be patients of LADA.

Studies related to the treatment regimen for patients with different types of diabetes revealed that almost all (92.5\%) patients with type 1 diabetes maintained optimum BSL with insulin. Majority of patients with type 2 diabetes $(65.5 \%)$ were treated with oral hypoglycemic agents (OHA) while a noticeable proportion $(29.5 \%)$ was on a combination of insulin and OHA suggesting development of progressive insulin dependence due to impairment in insulin secretion by various mechanisms [21,23]. Figure 5 depicts the results of statistical analysis on treatment regimen for different diabetic patients.

One of the major characteristics of LADA patients is initial response to OHA treatment [6]. Insulin dependency develops within a few years with gradual $\beta$-cell destruction necessitating a combined therapeutic regimen. In agreement with the reported literature, a major proportion of LADA patients $(60 \%)$ were found to be taking a combination of insulin and OHA. The observation would help in designing an effective therapeutic regimen for LADA patients, that is, a combined treatment should be prescribed at the time of diagnosis of LADA to preserve the remaining $\beta$-cell mass though it is still unclear whether early treatment with insulin is beneficial for the remaining $\beta$-cells [9]. Additionally, an initial response to OHA with gradual insulin dependency 


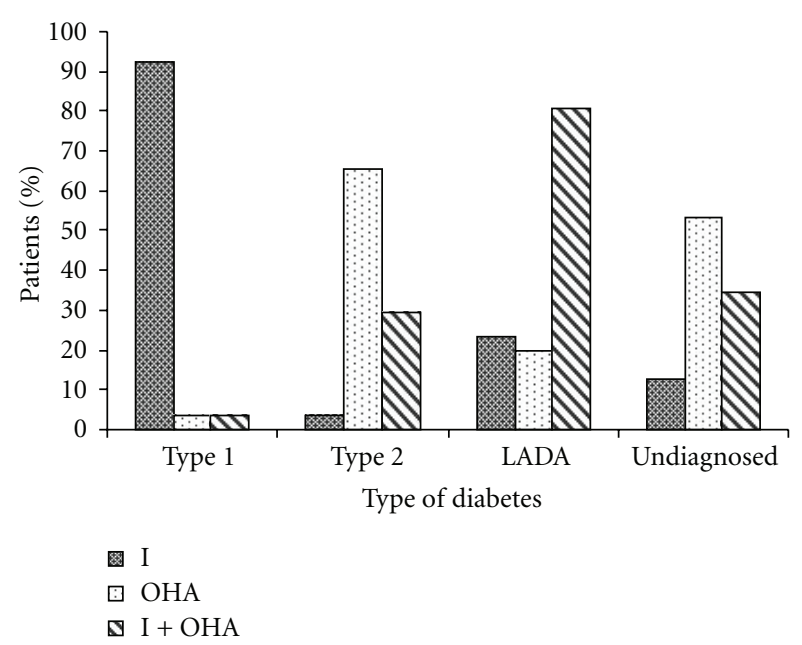

Figure 5: Treatment regimen for different types of diabetes. IInsulins, OHA-Oral Hypoglycemic Agents.

observed in patients younger than the usual age occurrence of type 2 diabetes would be a strong identifying characteristic for LADA patients. Thus the type of treatment that patients comply to, the most, can be predictive in different types of diabetes, specifically, LADA. A majority of the undiagnosed patients (53.5\%) were found to be on OHA, while a noticeable proportion (34.5\%) was found to be on a combined treatment. The proportion of patients under combined treatment $(34.5 \%)$ was relatively more than that of patients with type 2 diabetes $(29.5 \%)$ on a combined treatment. The numbers of undiagnosed patients on OHA alone were less than that with type 2 diabetes, while the number of patients on insulin and OHA was more than that with type 2 diabetes. This strongly suggests that these are likely to be LADA patients who initially respond to OHA, but insulin dependency may occur within some time and the number of patients on a combined treatment may increase in near future.

Furthermore, a detailed study about the drug therapy distribution in different types of diabetic patients was performed and patients were evaluated for the type of therapy being given, that is, monodrug therapy or multidrug therapy. Almost all patients with type 1 diabetes (96.4\%) showed optimum BSL with exclusive insulin monotherapy. No clear indication was observed for the remaining types of diabetes indicating that optimum BSL were maintained by a multidrug therapy (insulin + OHA or a combination of various OHA).

The HbAlc levels determination in diabetes gives an idea about the glycemic control during the last three months. Generally values above normal values (5-7\%) indicate a poor glycemic control [24]. HbA1c levels determined in patients with different types of diabetes showed that LADA patients (10-11\%) had significantly higher levels compared to type 1 $(9-10 \%)$ and type 2 diabetes $(8-8.5 \%)(P<0.05)$. This was in agreement with the published data [23]. This shows poorer glycemic control in LADA patients compared to type 1 and type 2 diabetes. Patients with type 1 diabetes showed relatively higher values as compared to patients with type 2 diabetes and lower than that of patients with LADA. Undiagnosed patients showed HbAlc values (7-8\%) similar to patients with type 2 diabetes. The values were higher than normal $\mathrm{HbAlc}$ levels $(5-7 \%)$ in all patients, indicating poor glycemic control in all patients [24].

Determination of serum cholesterol levels revealed that LADA patients $(200-250 \mathrm{mg} / \mathrm{dL})$ had significantly higher cholesterol levels than patients with type 2 diabetes (180$190 \mathrm{mg} / \mathrm{dL}$ ), which was found to be in accordance with existing data [4]. This indicates that LADA patients are at a higher risk of developing cardiovascular complications than patients with type 2 diabetes. No data was available for patients with type 1 diabetes. Interestingly, undiagnosed patients also displayed cholesterol values greater than that of patients with type 2 diabetes suggesting possible categorization of these patients as LADA patients.

C-peptide is secreted at equimolar concentrations with insulin and is not degraded as rapidly as insulin. Hence determination of C-peptide is an advantageous test to quantify insulin and therefore to evaluate $\beta$-cell function. Since LADA is an autoimmune type of diabetes characterized by progressive $\beta$-cell destruction, estimation of C-peptide levels would prove to be an important measure to evaluate insulin secretion and $\beta$-cell function. The results of the C-peptide determination showed significantly low levels of C-peptide in LADA patients $(0.34 \pm 0.05 \mathrm{ng} / \mathrm{mL})$ compared to patients with type 2 diabetes $(1.26 \mathrm{ng} / \mathrm{mL})(P<0.05)$. This is in accordance with existing data [6]. Many other studies suggested that LADA (also known as Ab positive type 2 diabetes) has lower C-peptide levels than patients with Ab negative type 2 diabetes [25-27]. Patients with type 1 diabetes also displayed lower C-peptide values $(0.36 \pm 0.05 \mathrm{ng} / \mathrm{mL})$ as compared to patients with type 2 diabetes. Low $\mathrm{C}$-peptide values in both the cases suggest insulin deficiency due to autoimmune $\beta$ cell destruction. Patients with type 2 diabetes displayed higher C-peptide values $(1.26 \pm 0.36 \mathrm{ng} / \mathrm{mL})$ which show hyperinsulinemia due to compensatory increase in insulin secretion due to insulin resistance in type 2 diabetes. C-peptide values of undiagnosed patients were found to be higher than that of patients with type 1 and LADA, but noticeably lower than that of patients with type 2 diabetes $(0.63 \pm 0.15 \mathrm{ng} / \mathrm{mL})$ suggesting that insulin deficiency could progress with time and lower C-peptiode values may be achieved at a later stage. This indicates that these patients are likely to be patients of LADA. Results are presented in Figure 6.

The immunological evidence, common in both type 1 diabetes and LADA, is demonstrated by the presence of autoantibodies against islet cell antigens in the patients' sera. Specifically, these antigens include $65 \mathrm{kDa}$ glutamic acid decarboxylase (GAD65) and insulinoma-associated antigen (IA2). While type 1 diabetes shows both these autoantibodies, LADA typically demonstrates production of GAD65 autoantibodies [14]. Hence estimation of GAD autoantibodies can be used as an important diagnostic marker for diagnosis of LADA. Since the test was qualitative in nature, GAD autoantibodies levels above 0.95 units $/ \mathrm{mL}$ indicated presence of GAD autoantibodies, while levels below this indicated absence of autoantibodies. Results of GAD autoantibodies determination revealed marked presence of GAD 


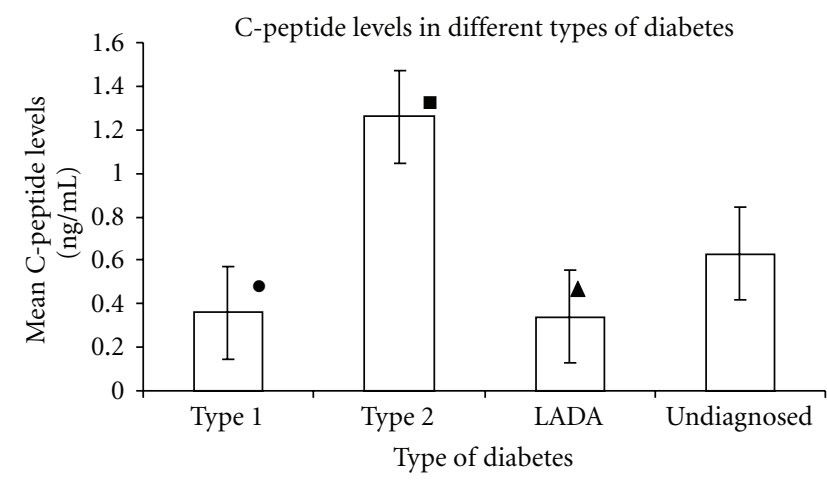

Figure 6: Mean C-peptide levels in different types of diabetes. Black circle: significantly lower C-peptide levels in type 1 diabetes as compared to type 2 diabetes $(P<0.05)$. Black square: significantly higher $\mathrm{C}$-peptide levels in type 2 diabetes as compared to type 1 diabetes and LADA $(P<0.05)$. Black triangle: significantly lower C-peptide levels in LADA as compared to type 2 diabetes $(P<0.05)$.

GAD auto antibodies in different types of diabetes

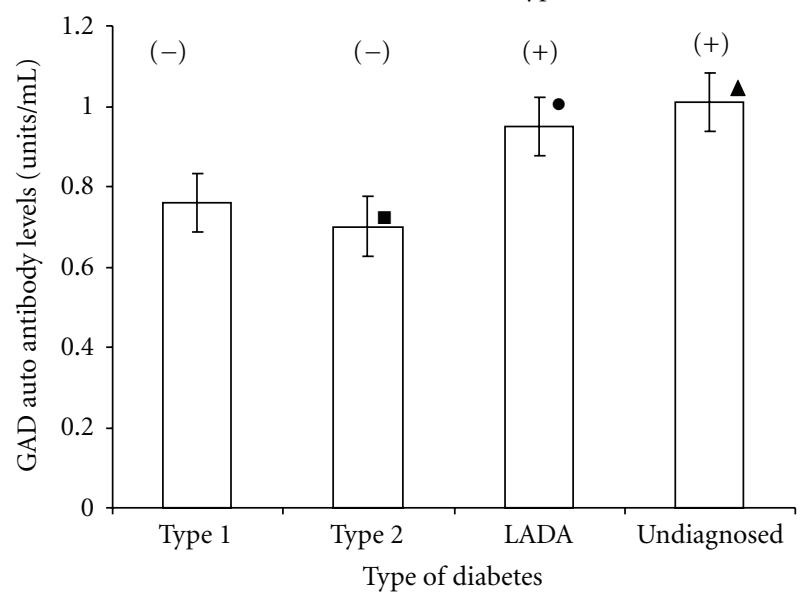

Figure 7: Mean GAD autoantibodies levels in different types of diabetes. Black square: significantly lower GAD autoantibodies levels in type 2 diabetes as compared to type 1 diabetes, LADA, and undiagnosed patients $(P<0.05)$. Black circle: significantly higher GAD autoantibodies levels in LADA as compared to type 1 diabetes and type 2 diabetes $(P<0.05)$. Black triangle: significantly higher GAD autoantibodies levels in undiagnosed patients as compared to type 1 diabetes $(P<0.01)$ and type 2 diabetes $(P<0.05)$. $(+)$ indicates presence of GAD auto antibodies. $(-)$ indicates absence of GAD auto antibodies.

autoantibodies predominantly in LADA patients $(0.95 \pm$ 0.06 units $/ \mathrm{mL})$ as compared to patients with type 2 diabetes $(P<0.05)$ (Figure 7$)$.

GAD autoantibodies were significantly present in undiagnosed patients $(1.01 \pm 0.07$ units $/ \mathrm{mL})$ as compared to patients with type 2 diabetes $(0.7 \pm 0.06$ units $/ \mathrm{mL})$ and type 1 diabetes $(0.76 \pm 0.05$ units $/ \mathrm{mL})$. This strongly depicts autoimmune nature of the disease, especially LADA, which is significantly in accordance with the existing data [6]. The characteristic presence of these antibodies in undiagnosed patients further strengthens the fact that these patients are most likely to be patients of LADA. The absence of GAD autoantibodies in type 2 diabetes implicates nonautoimmune nature of the disease $[28,29]$. The absence of these autoantibodies in type 1 diabetes do not imply that the disease is nonautoimmune in nature, but merely suggests that other autoantibodies like ICA, IA2, or tyrosine phosphatase autoantibodies may be involved $[30,31]$.

\section{Conclusions}

LADA patients comprise an important section of the diabetic population, its prevalence being nearly equal as that of type 1 diabetes. It can be characterized predominantly by adult age at onset (30-40 years), nonobese body type, and initial response to OHA gradually leading to insulin dependency, characteristically low C-peptide levels, and marked presence of GAD autoantibodies. Thus determination of C-peptide levels and GAD autoantibodies is strongly recommended for confirmatory diagnosis of LADA. The characteristic presence of GAD autoantibodies in LADA implies that further studies that identify possible role of GAD autoantibodies in LADA may help in understanding the pathogenesis of the disease. Interestingly the study showed that LADA seems to affect males more than females. LADA patients have also shown significant presence of family history, further exploration of which would provide newer insights into the role of family history as an important risk factor in development of LADA. Furthermore, genotyping of LADA patients will prove to be substantial in understanding the role of various genes involved in the disease. In conclusion, it appears that, while some anthropologic characteristics can be useful for the preliminary screening of LADA patients in a diabetic population, C-peptide levels and GAD autoantibodies determination can be considered as confirmatory diagnostic markers for LADA. Appropriate diagnosis of LADA would prevent misdiagnosis as type 2 diabetes and would help in optimum treatment of LADA patients so that residual $\beta$-cell function is preserved and the further autoimmune destruction of $\beta$ cells is delayed. For further information, see Supplementary Material available online at doi:10.5402/2012/580202.

\section{Acknowledgment}

Authors acknowledge All India Council for Technical Education, New Delhi, India, for financial support through NAFETIC scheme.

\section{References}

[1] J. M. Diamond, "Diabetes running wild," Nature, vol. 357, no. 6377, pp. 362-363, 1992.

[2] P. Z.Zimmet, "The pathogenesis and prevention of diabetes in adults: genes, autoimmunity, and demography," Diabetes Care, vol. 18, no. 7, pp. 1050-1064, 1995.

[3] R. D. Leslie and C. Valeri, "Latent autoimmune diabetes in adults," Diabetes Voice, vol. 48, no. 4, pp. 39-42, 2003.

[4] B. Isomaa, P. Almgren, M. Henricsson et al., "Chronic complications in patients with slowly progressing autoimmune type 1 diabetes (LADA)," Diabetes Care, vol. 22, no. 8, pp. 1347-1353, 1999. 
[5] World Health Organization, Definition, Diagnosis and Classification of Diabetes Mellitus and its Complications. Report of a WHO Consultation, WHO, Geneva, Switzerland, 1999.

[6] P. Zimmet, R. Turner, D. McCarty, M. Rowley, and I. Mackay, "Crucial points at diagnosis: type 2 diabetes or slow type 1 diabetes," Diabetes Care, vol. 22, supplement 2, pp. B59-B64, 1999.

[7] P. Pozzilli and U. Di Mario, "Autoimmune diabetes not requiring insulin at diagnosis (latent autoimmune diabetes off the adult): definition, characterization, and potential prevention," Diabetes Care, vol. 24, no. 8, pp. 1460-1467, 2001.

[8] A. Falorni and F. Calcinaro, "Autoantibody profile and epitope mapping in latent autoimmune diabetes in adults," Annals of the New York Academy of Sciences, vol. 958, pp. 99-106, 2002.

[9] M. Landin-Olsson, "Latent autoimmune diabetes in adults," Annals of the New York Academy of Sciences, vol. 958, pp. 112116, 2002.

[10] L. Wolfe, 2006, http://www.isletsofhope.com.

[11] S. Carlsson, K. Midthjell, and V. Grill, "Influence of family history of diabetes on incidence and prevalence of latent autoimmune diabetes of the adult: results from the Nord-Trøndelag health study," Diabetes Care, vol. 30, no. 12, pp. 3040-3045, 2007.

[12] K. G. Alberti and P. Z. Zimmet, "Definition, diagnosis and classification of diabetes mellitus and its complications. Part 1: diagnosis and classification of diabetes mellitus. Provisional report of a WHO consultation," Diabetic Medicine, vol. 15, no. 7, pp. 539-553, 1998.

[13] J. A. Scarlett, M. E. Mako, A. H. Rubenstein et al., "Factitious hypoglycemia. Diagnosis by measurement of serum C-peptide immunoreactivity and insulin-binding antibodies," The New England Journal of Medicine, vol. 297, no. 19, pp. 1029-1032, 1977.

[14] M. J. Clare-Salzler, A. J. Tobin, and D. L. O. Kaufman, "Glutamate decarboxylase: an autoantigen in IDDM," Diabetes Care, vol. 15, no. 1, pp. 132-135, 1992.

[15] S. Wild, G. Roglic, A. Green, R. Sicree, and H. King, "Global prevalence of diabetes: estimates for the year 2000 and projections for 2030," Diabetes Care, vol. 27, no. 5, pp. 1047-1053, 2004.

[16] H. Basavanagowdappa, A. K. Prabhakar, P. Prasannaraj, K. C. Gurudev, and V. Suma, "Study of prevalence of diabetes mellitus and impaired fasting glucose in a rural population," International Journal of Diabetes in Developing Countries, vol. 25, no. 4, pp. 98-101, 2005.

[17] S. Fourlanos, F. Dotta, C. J. Greenbaum et al., "Latent autoimmune diabetes in adults (LADA) should be less latent," Diabetologia, vol. 48, no. 11, pp. 2206-2212, 2005.

[18] R. Turner, I. Stratton, V. Horton et al., "UKPDS 25: autoantibodies to islet-cell cytoplasm and glutamic acid decarboxylase for prediction of insulin requirement in type 2 diabetes," The Lancet, vol. 350, no. 9087, pp. 1288-1293, 1997.

[19] T. Tuomi, Å. Carlsson, H. Li et al., "Clinical and genetic characteristics of type 2 diabetes with and without GAD antibodies," Diabetes, vol. 48, no. 1, pp. 150-157, 1999.

[20] H. A. Castleden, B. Shields, P. J. Bingley et al., "GAD antibodies in probands and their relatives in a cohort clinically selected for Type 2 diabetes," Diabetic Medicine, vol. 23, no. 8, pp. 834838, 2006.

[21] American Diabetes Association, "Diagnosis and classification of diabetes mellitus," Diabetes Care, vol. 29, 1, pp. S43-S48, 2006.
[22] B. Mlinar, J. Marc, A. Janež, and M. Pfeifer, "Molecular mechanisms of insulin resistance and associated diseases," Clinica Chimica Acta, vol. 375, no. 1-2, pp. 20-35, 2007.

[23] G. Biesenbach, M. Auinger, M. Clodi et al., "Prevalence of LADA and frequency of GAD antibodies in diabetic patients with end-stage renal disease and dialysis treatment in Austria," Nephrology Dialysis Transplantation, vol. 20, no. 3, pp. 559565, 2005.

[24] B. Panunti, A. A. Jawa, and V. A. Fonseca, "Mechanisms and therapeutic targets in type 2 diabetes mellitus," Drug Discovery Today, vol. 1, no. 2, pp. 151-157, 2004.

[25] American Diabetic Association, "Standards of medical care in diabetes-2007," Diabetes Care, vol. 30, 1, no. S4, p. S41, 2007.

[26] L. Groop, G. F. Bottazzo, and D. Doniach, "Islet cell antibodies identify latent type I diabetes in patients aged 35-75 years at diagnosis," Diabetes, vol. 35, no. 2, pp. 237-241, 1986.

[27] A. Kasuga, T. Maruyama, Y. Ozawa et al., "Antibody to the $\mathrm{Mr}$ 65,000 isoform of glutamic acid decarboxylase are detected in non-insulin-dependent diabetes in Japanese," Journal of Autoimmunity, vol. 9, no. 1, pp. 105-111, 1996.

[28] A. Gottsater, M. Landin-Olsson, A. Lernmark, P. Fernlund, and G. Sundkvist, "Islet cell antibodies are associated with $\beta$ cell failure also in obese adult onset diabetic patients," Acta Diabetologica, vol. 31, no. 4, pp. 226-231, 1994.

[29] R. A. DeFronzo, "Pathogenesis of type 2 (non-insulin-dependent) diabetes mellits: a balanced overview," Diabetologia, vol. 35, no. 4, pp. 389-397, 1992.

[30] D. Porte Jr., "Banting lecture 1990. Beta-cells in type II diabetes mellitus," Diabetes, vol. 40, pp. 166-180, 1991.

[31] E. F. Lampeter, M. Homberg, K. Quabeck et al., "Transfer of insulin-dependent diabetes between HLA-identical siblings by bone marrow transplantation," The Lancet, vol. 341, no. 8855, pp. 1243-1244, 1993. 

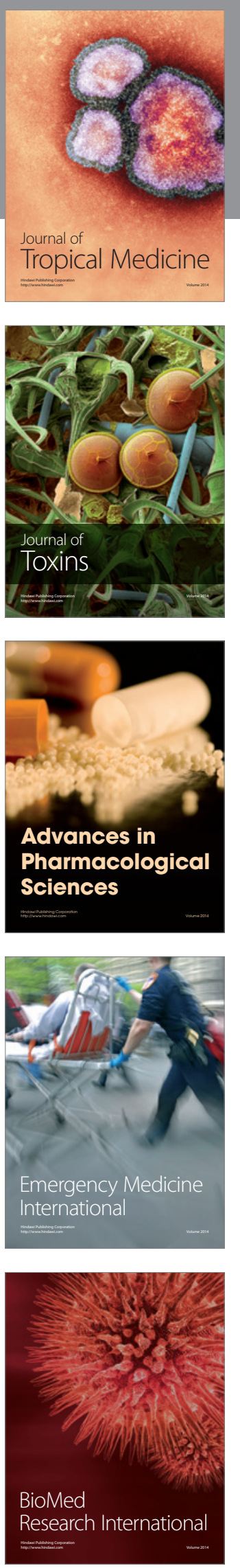
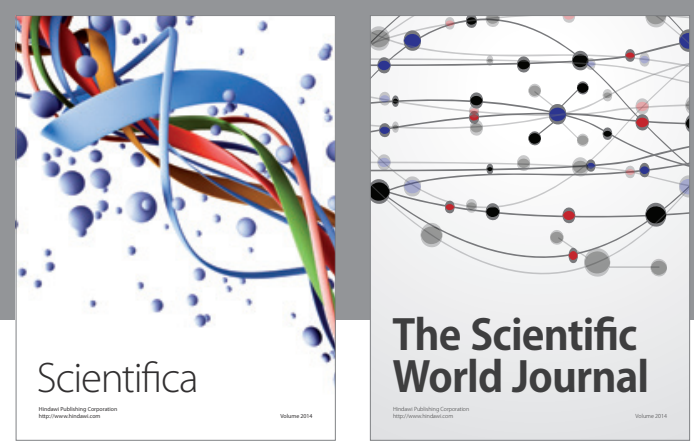

The Scientific World Journal
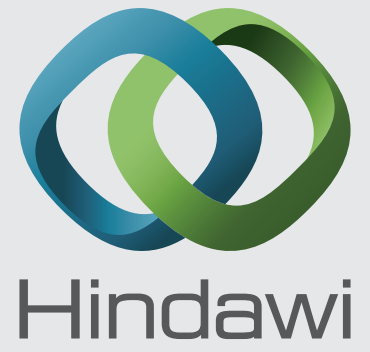

Submit your manuscripts at

http://www.hindawi.com
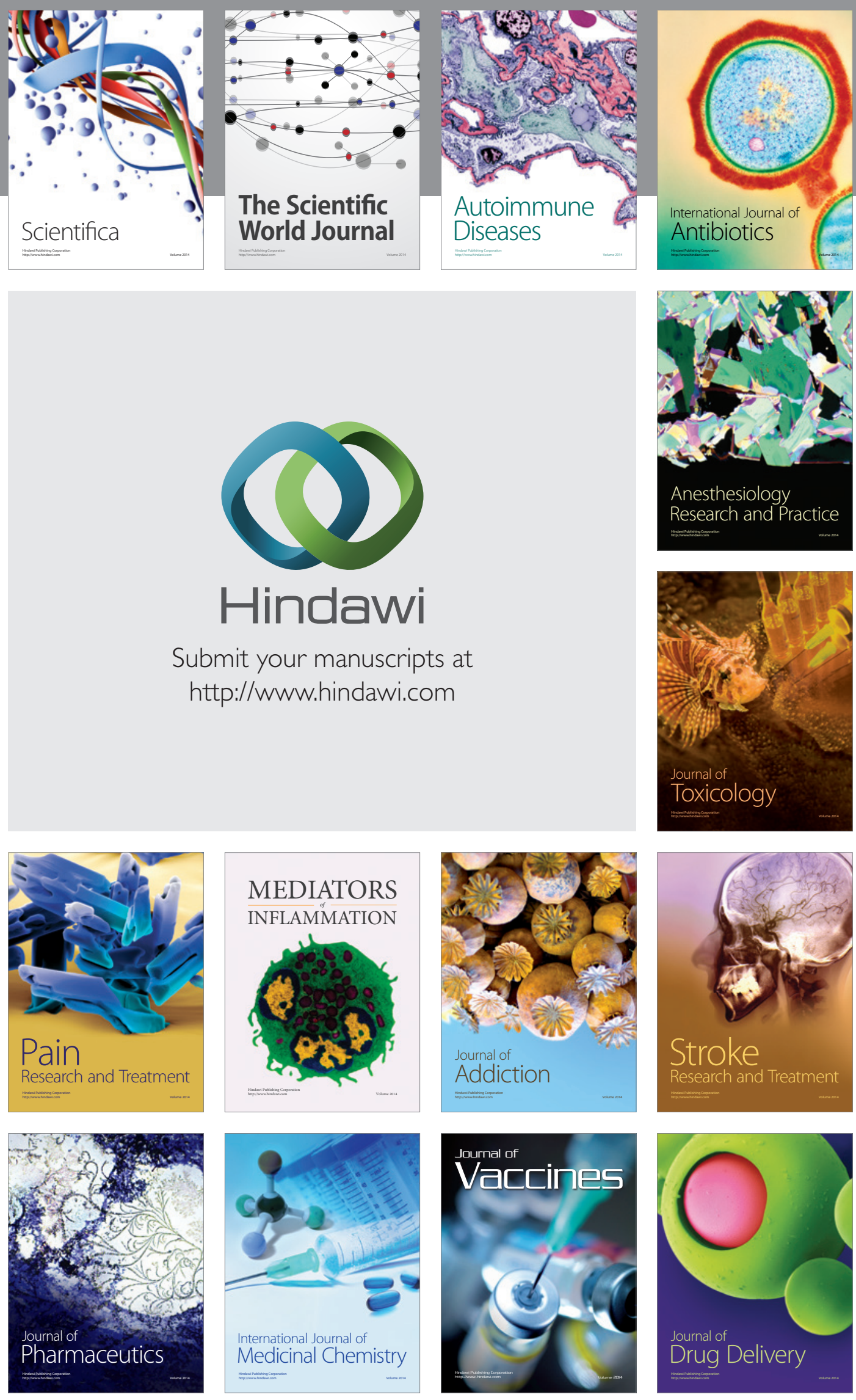